\title{
MANY TOPOLOGICAL ABELIAN GROUPS HAVE DENSE DIVISIBLE SUBGROUPS
}

\author{
R. C. HOOPER
}

1. Introduction. If $G$ is a complete, metrizable, topological abelian group such that the groups

$$
G^{m}=\{x \in G \mid m y=x \quad \text { for some } y \in G\}
$$

are dense in $G$, then Theorem 2.1 of this paper shows that $G$ has a dense divisible subgroup $K$. An example appears in $\$ 3$ of a group $G$ that is not divisible even though $G$ has a dense divisible subgroup. Also some of the possibilities are examined when $G$ is itself divisible. It should be remembered that the set of all divisible elements in an abelian group forms a subgroup.

2. The existence of dense divisible subgroups. Let $G$ be a complete, metrizable, topological abelian group. Define $G^{m}=\{x \in G \mid m y=x$ for some $y \in G\}$. Suppose that $G^{m}$ is dense in $G$ for all $m>0$.

Let $\left\{U_{n}\right\}_{n=1}^{\infty}$ be an open basis of the identity of $G$ such that $U_{n+1}+U_{n+1} \subset U_{n}$. Choose an open neighborhood $V_{n}$ of the identity of $G$ such that

$$
\begin{aligned}
& V_{n}+\cdots+V_{n} \subset U_{n} . \\
& \quad(n ! \text { times })
\end{aligned}
$$

Next a sequence $\left\{x_{i}\right\}_{i=1}^{\infty}$ will be defined inductively. Given $x \in G$, we suppose $x_{i}, 1 \leqq i \leqq n$ are given with $x_{1}=x$ and $\left((j+1) x_{j+1}-x_{j}\right) \in V_{j+1}$ whenever $x_{j}, x_{j+1}$ are defined. Define $x_{n+1}$ so that $\left((n+1) x_{n+1}-x_{n}\right)$ $\in V_{n+1}$. We can choose such an $x_{n+1}$ since $G^{n+1}$ is dense in $G$. For each $n$ consider the sequence $\left\{y_{n, m}\right\} \substack{\infty \\ m=n+1}^{\infty}$ where

$$
y_{n, m ; m>n}=(m)(m-1) \cdots(n+1) x_{m} .
$$

The claim is that $\left\{y_{n, m}\right\}_{m=n+1}^{\infty}$ is a Cauchy sequence. For consider

$$
\begin{gathered}
y_{n, m+1}-y_{n, m}=(m+1)(m) \cdots(n+1) x_{m+1}-(m)(m-1) \cdots(n+1) x_{m} \\
=(m)(m-1) \cdots(n+1)\left[(m+1) x_{m+1}-x_{m}\right] \in(m)(m-1) \cdots(n+1) \\
V_{m+1} \subset U_{m+1} .
\end{gathered}
$$

Thus $\left\{y_{n, m}\right\}_{m=n+1}^{\infty}$ forms a Cauchy sequence since $U_{m+1}+U_{m+1} \subset U_{m}$.

Let $y_{n}$ be the element which $\left\{y_{n, m}\right\}_{m}$ converges to. The claim is that $(n+1) y_{n+1}=y_{n}$. Given $U_{j}$, we may choose $m$ so that $y_{n+1, m}$ and

Received by the editors March 4, 1969. 
$y_{n, m}$ satisfy $y_{n+1, m}-y_{n+1} \in U_{j+n+2}$ and $y_{n, m}-y_{n} \in U_{j+1}$. Thus by the definition of $y_{n, m}$ and $y_{n+1, m},(m)(m-1) \cdots(n+2) x_{m}-y_{n+1} \in U_{j+n+2}$ and $(m)(m-1) \cdots(n+1) x_{m}-y_{n} \in U_{j+1}$. Hence

$$
\begin{aligned}
& (n+1) y_{n+1}-y_{n}=(n+1) y_{n+1}-(m)(m-1) \cdots(n+1) x_{m} \\
& +(m)(m-1) \cdots(n+1) x_{m}-y_{n} \in U_{j+1}+U_{j+1} \subset U_{j} .
\end{aligned}
$$

Since this is true for all $j$, we have $(n+1) y_{n+1}=y_{n}$.

As a consequence $n ! y_{n}=y_{1}$ for all $n$ and $y_{1}$ is divisible for all $n$.

Furthermore,

$$
\begin{aligned}
y_{1}-x= & \left(y_{1}-y_{1, m}\right)+\left(y_{1, m}-y_{1, m-1}\right)+\left(y_{1, m-1}-y_{1, m-2}\right)+\cdots \\
& +\left(y_{1,3}-y_{1,2}\right)+\left(y_{1,2}-x\right) \in U_{1}+U_{m}+U_{m-1}+\cdots \\
& +U_{3}+U_{2} \subset U_{1}+U_{1}
\end{aligned}
$$

since for any $j \geqq n, y_{n, j+1}-y_{n, j} \in U_{j+1}, y_{1,2}=2 x_{2}, y_{1,2}-x=2 x_{2}-x_{1} \in U_{2}$ and we may choose $m$ so that $y_{1}-y_{1, m} \in U_{1}$.

What this shows since $U_{1}$ can be chosen as small a neighborhood as desired and since $x$ was arbitrary, is that the divisible elements are dense in $G$.

THEOREM 2.1. A complete, metrizable topological abelian group $G$ has a dense divisible subgroup if and only if the $G^{m}$ are dense in $G$ for all $m>0$.

Proof. If the $G^{m}$ are dense, then it was just shown that $G$ has a dense divisible subgroup. On the other hand if $G$ has a dense divisible subgroup $H, G^{m} \cap H=H$ is dense in $G$.

The next theorem gives one example of groups which have a dense divisible subgroup.

THEOREM 2.2. Let $G$ be a topological abelian group. If $G$ has a dense, cyclic subgroup $H$ and $G$ has no proper open subgroups, then the subgroups $G^{n}$ are dense for all $n>0$.

Proof. Suppose one of the $G^{n}$ for $n>0$ is not dense in $G$. Then if $H_{1}=G^{n} \cap H$, let $H_{2}$ be the closure of $H_{1}$ in $H$. Since $H$ is algebraically isomorphic to the integers and $\mathrm{H}_{2}$ is not the zero subgroup, $\mathrm{H}_{2}$ is open in $H$. Hence since $H$ is dense in $G$, the closure $L$ of $H_{2}$ in $G$ is an open subgroup of $G$. But since we supposed that $G^{n}$ is not dense in $G$, $L$ would be a proper open subgroup of $G$ which is a contradiction.

A special class of groups like those of Theorem 2.1 are those which are divisible as well as being complete and metrizable. If $H$ is a divisible group such that the map $\phi_{1 / n}: x \rightarrow(1 / n) x$ is well defined and a continuous homomorphism for all $x \in H$, then the map $\phi_{1 / n}$ extends to a continuous homomorphism of the completion $G$ of $H$. Naturally 
such a $G$ must be torsion free. Any topological vector space is one example of such a group.

Suppose on the other hand $G$ is complete, metrizable, separable, and divisible. Then $\phi_{n}: G \rightarrow G$ is continuous and onto. Thus by the open mapping theorem $\left[1\right.$, p. 99], $\phi_{n}$ is open.

If $G$ is also torsion free, then the mapping $\phi_{1 / n}: x \rightarrow(1 / n) x$ is well defined and is a homomorphism. Since $\phi_{n}$ is open, $\phi_{1 / n}=\phi_{n}^{-1}$ is continuous. The solenoidal group $\Sigma_{a}, \mathrm{a}=(1,2,3, \cdots)[2$, p. 406 and p. 388 ] is one example of such a torsion free group $G$. The $a$-dic solenoid $\Sigma_{a}$ is compact, connected, divisible, and torsion free. But even though the mappings $\phi_{1 / n}$ are continuous for all $n>0, \Sigma_{a}$ is not a topological vector space.

The circle group is an example of a connected, complete, metrizable divisible group such that the mappings $\phi_{1 / n}$ are not well defined for any $\boldsymbol{n}>0$.

3. The size of the maximal divisible subgroup of $G$. If $G_{1}$ and $G_{2}$ are topological groups and $\phi: G_{1} \rightarrow G_{2}$ is a homomorphism, then $\phi$ is called almost open [1, p. 14 and p. 89] if for every $x \in G, y=\phi(x)$, and neighborhood $U$ of $x$, the closure of $\phi(U), \mathrm{Cl}(\phi(U))$, is a neighborhood of $\phi(y)$. If $\phi: G_{1} \rightarrow G_{2}$ is almost open and $H_{1}$ is a dense subgroup of $G_{1}$, then it can be checked that $\phi$ restricted to $H_{1}$ is almost open.

Suppose that we are given an almost open homomorphism $\phi^{\prime}: H_{1} \rightarrow H_{2}$. If $H_{1}$ is dense in $G_{1}, H_{2}$ is dense in $G_{2}$, and $\phi: G_{1} \rightarrow G_{2}$ is an extension of $\phi^{\prime}$ to $G_{1}$, then it can be checked that $\phi$ is almost open.

Even if the $G^{m}$ are dense in $G$, the maps $\phi_{n}: G \rightarrow G$ do not have to be almost open. To see this, first define on the additive integers a topology called the group series topology. Let a set $S=\left\{\left(p_{i}, M_{i}\right)\right\}_{i=1}^{\infty}$ of pairs of positive integers be given such that $M_{i}=2^{i}$, the greatest common divisor of $\left\{p_{i}\right\}_{i=n}^{\infty}$ is 1 for all $n$, and such that $3 \sum_{i=1}^{j-1} M_{i}<p_{j}$ for all $j$. Define

$$
U_{n}=\left\{x \mid x=\sum_{i=n}^{N} m_{i} p_{i} \text { for }\left|m_{i}\right| \leqq 2^{--n} M_{i}, \quad \text { for } m_{i} \text { and } N \text { integers }\right\} .
$$

These $U_{n}$ satisfy the axioms $[1$, p. 46] for a basis of the neighborhoods of the identity for a topological abelian group, i.e., $U_{n}=-U_{n}$, and $U_{n+1}+U_{n+1} \subset U_{n}$. For if $x, y \in U_{n+1}$, then $x=\sum_{i=1}^{N} m_{i} p_{i}$ and $y=\sum_{i=1}^{N} m_{i}^{\prime} p_{i}$ for some integers $m_{i}, m_{i}^{\prime}, N$ such that $\left|m_{i}\right| \leqq\left(1 / 2^{n+1}\right)$ $M_{i},\left|m_{i}^{\prime}\right| \leqq\left(1 / 2^{n+1}\right) M_{i}$. Thus $x+y$ can be written as $x+y=\sum_{i=1}^{N}$ $\left(m_{i}+m_{i}^{\prime}\right) p_{i}$ with $\left|m_{i}+m_{i}^{\prime}\right| \leqq\left(1 / 2^{n}\right) M_{i}$. This proves that $x+y \in U_{n}$ and as a result $U_{n+1}+U_{n+1} \subset U_{n}$.

If $x \in U_{n}$, then $x=\sum_{i=1}^{N} m_{i} p_{i}$ for some integers $N, m_{i}$ such that $\left|m_{i}\right| \leqq\left(1 / 2^{n}\right) M_{i}$. Thus $-x=\sum_{i=1}^{N} \quad\left(-m_{i}\right) p_{i}$ and $\left|-m_{i}\right|=\left|m_{i}\right|$ 
$\leqq\left(1 / 2^{n}\right) M_{i}$. Whence $-x \in U_{n}$ and therefore $-U_{n}=U_{n}$.

Furthermore if $x \in U_{1}$, then there is a unique set of integers $\left\{m_{i}\right\}_{i}$ with $x=\sum_{i}^{N} m_{i} p_{i}$ and $\left|m_{i}\right| \leqq M_{i}$. For if not for some $m_{i}, m_{i}^{\prime}, N$, and $j$, $x=\sum_{i=1}^{N} m_{i} p_{i}=\sum_{i=1}^{N} m_{i}^{\prime} p_{i}$ with $\left|m_{i}\right| \leqq M_{i},\left|m_{i}^{\prime}\right| \leqq M_{i}$ and $j$ the largest index such that $m_{j} \neq m_{j}^{\prime}$. Then $\left|\left(m_{j}-m_{j}^{\prime}\right) p_{j}\right|=\mid \sum_{i=1}^{j-1}$ $\left(m_{i}-m_{i}^{\prime}\right) p_{i} \mid \leqq \sum_{i=1}^{j-1} 2 M_{i}$. But since the $p_{i}$ were chosen so that $p_{j}>3 \sum_{i=1}^{j-1} M_{i}$, then we have $\left|\left(m_{j}-m_{j}^{\prime}\right) p_{j}\right|>3 \sum_{i=1}^{j-1} M_{i}$ which is a contradiction. Henceforth if we write $x=\sum_{i} m_{i} p_{i}$, it will be assumed that $\left|m_{i}\right| \leqq M_{i}$. Since the greatest common divisor of the elements of any $U_{n}$ is 1 , then the smallest subgroup of the integers containing any $U_{n}$ is the integers. Thus if the topological group $H$ is the integers under the topology having a basis of the identity the $U_{n}, H$ has no proper open subgroups and $H^{m}$ is dense in $H$ for all $m>0$.

If $\phi_{2}: H \rightarrow H$ is defined by $\phi_{2}: x \rightarrow 2 x$, then the claim is that $\mathrm{Cl}$ $\left(\phi_{2}\left(U_{3}\right)\right)$ ls not a neighborhood of 0 . If $\mathrm{Cl}\left(\phi_{2}\left(U_{3}\right)\right)$ is a neighborhood of $O$, then we can find some $U_{j}$ with $j \geqq 3$ such that $U_{j} \subset \mathrm{Cl}\left(\phi_{2}\left(U_{3}\right)\right)$.

In particular $p_{j} \in \mathrm{Cl}\left(\phi_{2}\left(U_{3}\right)\right)$ and there is a $c \in \phi_{2}\left(U_{3}\right)$ such that $p_{j}-c \in U_{j+1}$. If $c=\sum_{i=1}^{N} n_{i} p_{i}$, all $n_{i}$ are divisible by 2 since $c \in \phi_{2}\left(U_{3}\right)$. Also

$$
p_{j}-c \in U_{j}+\phi_{2}\left(U_{3}\right) \subset U_{2}+U_{2} \subset U_{1}
$$

implies that we can write $p_{j}-c=\sum_{i=1}^{N^{\prime}} q_{i} p_{i}$ with the $q_{i}$ unique. Since 2 divides $n_{j}, q_{j}=1-n_{j}$ is not zero. This contradicts the fact that $p_{j}-c \in U_{j+1}$. Hence $\mathrm{Cl}\left(\phi_{2}\left(U_{3}\right)\right)$ is not a neighborhood of 0 and $\phi_{2}$ is not almost open.

Let $G$ be the completion of $H$, the integers, under this topology and let $K$ be the maximal divisible group of $G$. $K$ is dense in $G$ by Theorem 2.1. Suppose $K=G$.

Let $\phi_{2}^{\prime}: x \rightarrow 2 x$ be the extension of $\phi_{2}$ to $G$. Since $G$ is divisible, $\phi_{2}$ is onto. But since $G$ is complete and $\phi_{2}^{\prime}$ is continuous and onto, then $\phi_{2}^{\prime}$ is an open mapping [1, p. 99]. But then since $\phi_{2}^{\prime}$ is almost open, the restriction $\phi_{2}$ of $\phi_{2}^{\prime}$ to $H$ is almost open. This is a contradiction. Hence $K \neq G$.

Thus we have an example of a complete, metrizable group $G$ such that even though the $G^{n}$ are dense, $G$ is not divisible. The author does not know whether $K$ is always uncountable when $G$ is uncountable.

\section{BIBLIOGRAPHY}

1. T. Husain, Introduction to topological groups, Saunders, Philadelphia, Penn., 1966.

2. E. Hewitt and K. Ross, Abstract harmonic analysis, Academic Press, New York, 1963.

UNIVERSITY OF NEVADA 